PACS: $29.20 . \mathrm{db}, 29.27 . \mathrm{Bd}, 29.27 . \mathrm{Fh}$

\title{
REDESIGN OF NESTOR STORAGE RING LATTICE
}

\author{
P.I. Gladkikh, A.A. Kalamaiko, I.M. Karnaukhov \\ National Scientific Center Kharkov Institute of Physics and Technology \\ 1, Akademicheskaya St., Kharkiv, 61108, Ukraine \\ E-mail: kalamaiko@kipt.kharkiv.ua \\ Received August 5, 2017
}

NESTOR is the hard X-ray source that is under commissioning at NSC KIPT. NESTOR based on the Compton scattering of laser photons on relativistic electrons. The structure of the facility can be represented as the following components: a linear accelerator, a transport channel, a storage ring, and a laser-optical system. Electrons are stored in the storage ring for energy of $40-225 \mathrm{MeV}$, laser photons are accumulated in the optical resonator. The frequencies of laser and electron bunches are synchronized, the interaction point located in the optical resonator. The measurements showed that parameters of the manufactured bending magnets different from the design ones. The bending magnets have different field index, besides the vertical focusing forces are significantly lower than designed ones. The beam dynamics simulation showed that due to decreasing of the vertical focusing the vertical betatron frequency becomes close to the frequency of the integer resonance $\mathrm{Q}=2$, as a result the vertical motion in the ring is unstable [1]. The second reason for optimization is the need to increase the length of the drift space for optical resonator. The results of optimization are presented in this paper.

KEYWORDS: compton scattering, storage ring, lattice redesign, beam dynamics simulation, dynamics aperture

\section{МОДЕРНИЗАЦИЯ СТРУКТУРЫ ФОКУСИРОВКИ НАКОПИТЕЛЬНОГО КОЛЬЦА НЕСТОР \\ П.И. Гладких, А.А. Каламайко, И.М. Карнаухов}

Национальный научный центр «Харьковский физико-технический институт»

61108, Украина, г. Харьков, ул. Академическая, 1.

В ННЦ ХФТИ сооружается источник жесткого излучения “НЕСТОР”, основанный на комптоновском рассеянии лазерных фотонов на релятивистских электронах. Структурно установка может быть представлена в виде следующих компонентов: линейный ускоритель, канал транспортировки, накопительное кольцо, лазерно-оптическая система. Электроны накапливаются в накопительном кольце на энергию 40-225 МэВ, лазерные фотоны накапливаются в оптическом резонаторе. Частоты следования лазерных и электронных сгустков синхронизированы, точка взаимодействия находится в оптическом резонаторе. Измерения показали, что параметры изготовленных поворотных магнитов отличаются от проектных. Поворотные магниты не только имеют разные показатели спада магнитного поля, но и силы вертикальной фокусировки существенно ниже проектных. Расчеты показали, что из-за уменьшения вертикальной фокусировки частота вертикальных бетатронных колебаний становится близкой к частоте целого резонанса $\mathrm{Q}=2$, в результате чего вертикальное движение в кольце становится неустойчивым [1]. Вторая причина модернизации заключается в необходимости увеличить длину одного из дрейфовых промежутков для установки оптического резонатора. Результаты модернизации представлены в данной работе.

КЛЮЧЕВЫЕ СЛОВА: комптоновское рассеяние, накопительное кольцо, модернизация структуры фокусировки, моделирование динамики пучка, динамическая апертура

\section{МОДЕРНІЗАЦІЯ СТРУКТУРИ ФОКУСУВАННЯ НАКОПИЧУВАЛЬНОГО КІЛЬЦЯ НЕСТОР \\ П.І. Гладких, А.А. Каламайко, І.М. Карнаухов \\ Національний науковий центр «Харківський фізико-технічний інститут» \\ 61108, Украӥна, м.Харків, вул Академічна, 1.}

В ННЦ ХФТІ споруджується джерело жорсткого випромінювання "НЕСТОР", засноване на комптонівському розсіюванні лазерних фотонів на релятивістських електронах. Структурно установка може бутипредставлена у вигляді наступних компонентів: лінійний прискорювач, канал транспортування, накопичувальне кільце, лазерно-оптична система. Електрони накопичуються в накопичувальному кільці на енергію 40-225 MeB, лазерні фотони накопичуються в оптичному резонаторі. Частоти проходження лазернихі електронних згустків синхронізовані, точка взаємодії знаходиться в оптичному резонаторі. Вимірювання показали, що параметри виготовлених поворотних магнітів відрізняються від проектних. Поворотні магніти не тільки мають різні показники спаду магнітного поля, а й сили вертикального фокусування істотно нижче проектних. Розрахунки показали, що через зменшення вертикальної фокусування частота вертикальних бетатронних коливань стає близькою до частоти цілого резонансу $\mathrm{Q}=2$, в результаті чого вертикальний рух в кільці стає нестійким [1]. Друга причина модернізації полягає в необхідності збільшити довжину одного 3 дрейфовий проміжків для установки оптичного резонатора. Результати модернізації представлені в даній роботі.

КЛЮЧОВІ СЛОВА: комптонівське розсіювання, накопичувальне кільце, модернізація структури фокусування, моделювання динаміки пучка, динамічна апертура

Идея генерации высокоэнергетичных фотонов на основе обратного комптоновского рассеяния была предложена в 1951 - 52 Х. Мотцем [2] и К. Ландекером [3]. Затем, метод обратного комптоновского рассеяния был развит в 1963 году Ф.Р. Арутюняном и В.А. Туманяном [4], а также независимо Мильбурном [5]. Но возможности ускорительной и лазерной техники в то время не позволяли рассматривать предложенный метод для практических целей из-за очень малого поперечного сечения процесса $\left(\sigma_{\mathrm{c}} \sim 6,6 \times 10^{-29} \mathrm{M}^{2}\right)$. 
В настоящее время достижения в области лазерной и ускорительной техники открывают новые возможности в генерации жесткого излучения высокой энергии на основе обратного комптоновского рассеяния лазерных фотонов на релятивистских электронах [6].

При однократном столкновении электронного и лазерного сгустков число рассеянных фотонов определяется светимостью L и полным сечением комптоновского рассеяния $\sigma$ [7]

$$
\mathrm{n}_{\gamma}=\mathrm{L} \sigma .
$$

В лабораторной системе отсчета выражение для светимости, в случае гауссовских распределений плотностей в электронных и лазерных сгустках, имеет следующий вид [8]

$$
\mathrm{L}=\frac{\mathrm{n}_{\mathrm{e}} \mathrm{n}_{1}}{2 \pi \sqrt{\left(\sigma_{\mathrm{ze}}^{2}+\sigma_{\mathrm{zl}}^{2}\right)} \sqrt{\left(\sigma_{\mathrm{xe}}^{2}+\sigma_{\mathrm{xl}}^{2}\right) \cos ^{2}(\varphi / 2)+\left(\sigma_{\mathrm{se}}^{2}+\sigma_{\mathrm{sl}}^{2}\right) \sin ^{2}(\varphi / 2)}},
$$

где $\sigma_{\mathrm{xe}}, \sigma_{\mathrm{xl}}, \sigma_{\mathrm{ze}}, \sigma_{\mathrm{zl}}$ - среднеквадратичные поперечные размеры в точке столкновения электронного и лазерного пучков, $\sigma_{\mathrm{se}}, \sigma_{\mathrm{sl}}$ - среднеквадратичные продольные размеры электронного и лазерного сгустков, $\mathrm{n}_{\mathrm{e}}, \mathrm{n}_{1}-$ число электронов и фотонов в сталкивающихся пучках, $\varphi$ - угол столкновения электронного и лазерного пучков.

Энергетический спектр рассеянных фотонов характеризуется максимальной энергией $\varepsilon_{\max }$, приобретаемой фотоном при лобовом столкновении [8]

где $\gamma$ - Лоренц - фактор, $\varepsilon_{1}-$ энергия лазерного фотона.

$$
\varepsilon_{\max }=4 \gamma^{2} \varepsilon_{1}
$$

Принимая во внимание все вышеизложенное, для получения максимальной интенсивности необходимо создать накопительное кольцо с минимально возможными поперечными и продольными размерами электронного и лазерного сгустков в точке столкновения. Кроме того, технология должна обеспечивать минимальный угол столкновения.

Основная проблема в разработке подобной структуры заключается в большой величине энергетического разброса в электронном пучке. При комптоновском рассеянии электрон теряет значительную часть своей энергии и, вследствие стохастичности этого процесса, это приводит к росту энергетического разброса [9]. В этих условиях, для получения устойчивого движения важно обеспечить большой энергетический аксептанс накопителя. Для получения большого аксептанса в накопительном кольце необходимо добиться малого значение коэффициента упаковки орбит, т.к. его величина определяется выражением [8]:

$$
\sigma_{\mathrm{rf}} \propto \sqrt{\frac{V_{R F}}{\alpha_{1}}},
$$

где $\sigma_{\mathrm{rf}}$ - энергетический аксептанс, $\mathrm{V}_{\mathrm{rf}}$ - ускоряющее ВЧ-напряжение, $\alpha_{1}$ - линейный коэффициент упаковки орбит.

Для описания поперечного смещения пучка при больших значениях отклонения импульса необходимо также учитывать квадратичную дисперсию [10]

$$
\Delta x=\eta_{1} \frac{\Delta p}{p}+\eta_{2}\left(\frac{\Delta p}{p}\right)^{2}
$$

где $\eta_{1}$ и $\eta_{2}$ - линейная и квадратичная дисперсия, $\frac{\Delta p}{p}$ - относительное отклонение импульса.

В случае больших значений отклонения импульса и квадратичной дисперсии, квадратичные члены начинают заметно влиять на удлинение орбиты, другими словами, коэффициент упаковки орбит становится зависимым от отклонения импульса [10].

$$
\alpha=\alpha_{1}+\alpha_{2} \frac{\Delta p}{p}
$$

Когда линейный коэффициент упаковки орбит становится меньше некоторого критического значения, энергетический аксептанс накопительного кольца начинает уменьшаться [11], поэтому линейный коэффициент упаковки орбит является некоторой компромиссной величиной.

Цель данной работы заключается в проведении модернизации системы фокусировки накопительного кольца НЕСТОР и оценки интенсивности жесткого излучения с учетом реальных параметров накопительного кольца и планируемых параметров оптической системы.

\section{ПРОЕКТНАЯ СХЕМА НАКОПИТЕЛЬНОГО КОЛЬЦА}

Основные требования к фокусирующей структуре накопителя следующие:

1. Как следует из (2), для получения высокой интенсивности комптоновского рассеяния необходимо обеспечить минимальные продольные и поперечные размеры электронного и лазерного сгустков в точке взаимодействия. Поперечные размеры электронного сгустка в точке взаимодействия определяются следующим 
выражением $\sigma_{x e, z e}=\sqrt{\varepsilon_{x, z} \beta_{x, z}}$, где $\varepsilon_{x, z}-$ эмитанс электронного пучка в горизонтальной (х) и вертикальной (z) плоскости, $\beta_{x, z}$ - амплитудные функции в точке взаимодействия в соответствующей плоскости. Поэтому необходимо обеспечить минимальное значение амплитудных функций в точке взаимодействия.

Продольный размер электронного сгустка $\sigma_{\mathrm{se}}$ пропорционален коэффициенту упаковки орбит $\sigma_{\mathrm{se}} \propto \sqrt{\alpha_{1} / V_{R F}}$, здесь $\mathrm{V}_{\mathrm{rf}}$ - ускоряющее ВЧ-напряжение, $\alpha_{1}$ - линейный коэффициент упаковки орбит. Таким образом, структура фокусировки должна обеспечить малое значение коэффициента упаковки орбит.

2. Количество секступольных линз должно быть достаточным для подавления хроматических эффектов и коррекции динамической апертуры.

3. Необходимо учитывать эффекты внутрипучкового рассеяния, так как на малых энергиях эти эффекты приводят к быстрой деградации электронного пучка - размеры сгустка в поперечной и продольной плоскости возрастают, что приводит к падению интенсивности рассеяния.

С учетом всего вышеизложенного была разработана схема накопительного кольца с контролируемым коэффициентом упаковки орбит (рис.1).

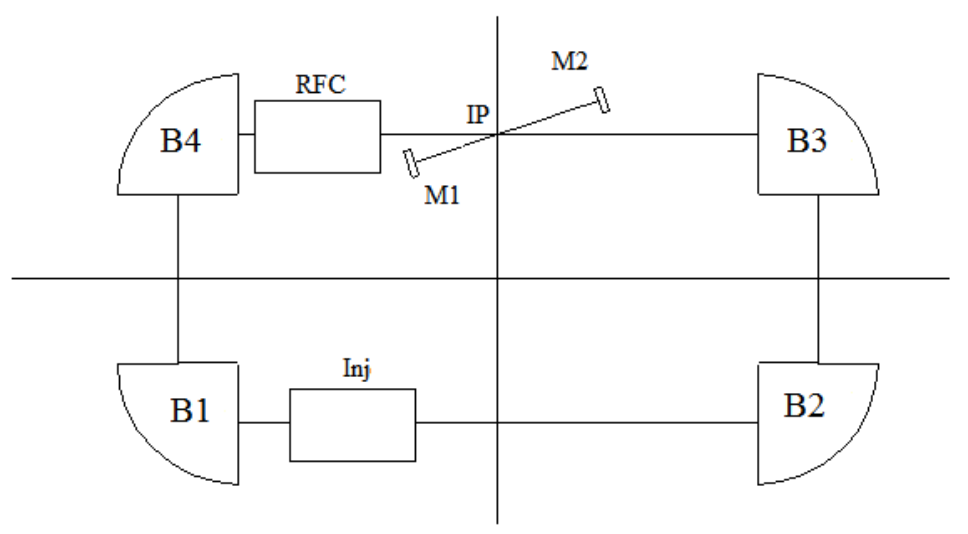

Рис. 1: Схема накопительного кольца НЕCTOP. IP - точка взаимодействия, B1-B4 - поворотные магниты, RFC резонатор ВЧ-подпитки, Inj - ударный магнит системы инжекции, M1-M2 - зеркала оптического резонатора.

Поворотная арка это часть структуры накопителя, ограниченная поворотными магнитами, которая осуществляет поворот пучка. Поворотные арки накопительного кольца НЕСТОР представляют собой систему, состоящую из двух поворотных магнитов с двумя квадрупольными линзами, расположенными между ними. Уменьшить коэффициент упаковки орбит можно, если сделать дисперсионную функцию отрицательной на одном из поворотных магнитов поворотной арки с помощью квадрупольных линз расположенных в ней. В этом случае одна длинная прямолинейная секция (B3-B4) будет с нулевой дисперсией, дисперсионная функция на противоположной прямолинейной секции (B1-B2) будет отлична от нуля. ВЧ-резонатор (RFC) и лазернооптическая система (M1-M2) размещаются в секции с нулевой дисперсией, система инжекции размещается на противоположной прямолинейной секции (Inj).

В настоящее время Nd:YAG - лазеры с энергией фотонов 1,16 эB считаются наиболее перспективными для использования в лазерно-электронных накопительных кольцах [12]. Частота следования импульсов в таких лазерах достигает сотен МГц, а средняя мощность около 10 Вт.

Примем для оценок интенсивности комптоновского рассеяния поперечные размеры лазерного пятна в точке взаимодействия равными 50 мкм, продольный размер 1,5 мм, частота следования лазерного импульса 350 МГц, средняя мощность $\mathrm{P} \approx 10 \mathrm{Bт}$, такой лазер излучает примерно 28 нДж $\left(1,5 \times 10^{11}\right.$ фотонов) в одном импульсе. Будем считать, что на орбите накопителя находится один электронный сгусток с зарядом 2 нКл, а размеры электронного и лазерного пучков совпадают. В этом случае за одну секунду будет сгенерировано примерно $3 \times 10^{7}$ фотонов.

Для увеличения интенсивности необходимо увеличивать число электронов на орбите (увеличивать число электронных сгустков) и увеличивать энергию лазерной вспышки, для чего используется оптический резонатор, в котором будет накапливаться излучение лазера. Все это позволяет достичь интенсивности жесткого излучения порядка $10^{13}-10^{14}$ фот/сек. При энергии электронного пучка в диапазоне от 40 до 225 МэВ, можно генерировать жесткое излучение с энергией от 30 до 900 КэВ.

\section{ПРОЕКТНАЯ СТРУКТУРА ФОКУСИРОВКИ НЕСТОР}

Проектная структура фокусировки накопительного кольца НЕСТОР [13] представлена на рис.2. Периметр ускорителя составляет 15,418 м. Поворотные магниты (B1-B4) имеют радиус поворота 0,5 м, угол поворота 
$90^{\circ}$, магнитное поле на максимальной рабочей энергии электронного пучка (225 МэВ) составляет 1,5 Тл. Для обеспечения фокусировки в вертикальной плоскости поворотные магниты имеют квадрупольную составляющую магнитного поля.

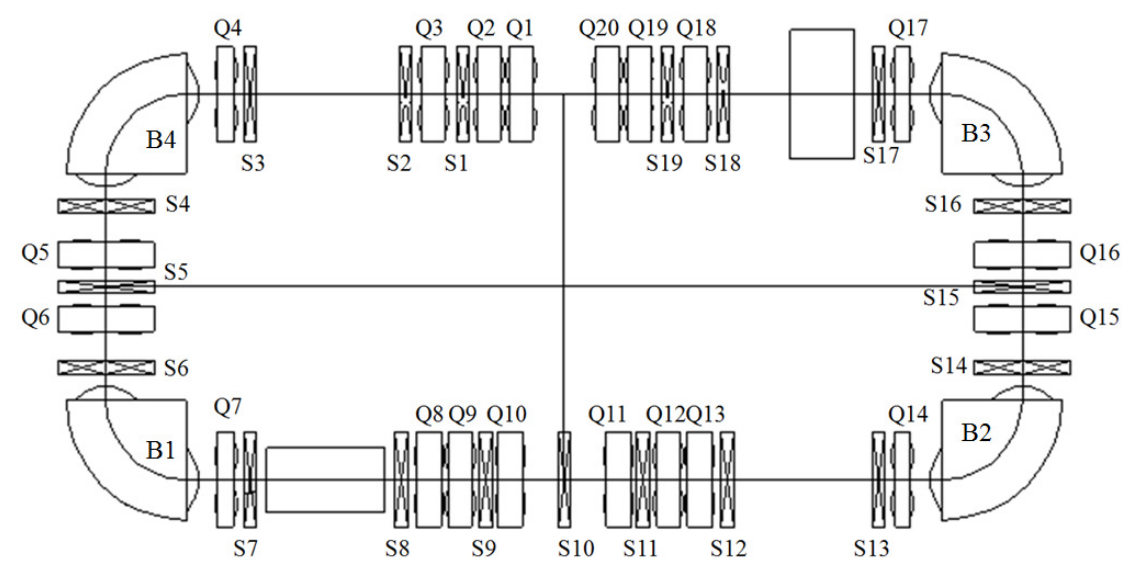

Рис.2: Структура фокусировки накопительного кольца НЕСТОР Q1 - Q20 - квадрупольные линзы, S1 - S19 - секступольные линзы

Промежуток инжекции (B1-В2) имеет ненулевую дисперсию $\eta_{1} \neq 0$, в то время как промежуток взаимодействия (B3-B4), содержащий оптический резонатор ахроматичен $\eta_{1}=0$, а в точке взаимодействия дисперсия второго порядка минимальна. С помощью квадрупольных линз (Q5, Q6, Q15, Q16) обеспечивается отрицательное значение дисперсионной функции $\eta_{1}$ на части траектории в поворотных арках (B4-B1, B2-B3), что позволяет получить малый коэффициент упаковки орбит $\alpha \approx 0,01$. Такое малое значение коэффициента упаковки орбит обеспечивает энергетический аксептанс накопителя на уровне $4 \%$.

Для фокусировки электронного пучка в точке взаимодействия используются квадруплеты квадрупольных линз (Q1-Q4, Q17-Q20). Такая схема дает возможность получить минимальные значения горизонтальной и вертикальной амплитудных функций в точке взаимодействия. Триплеты (Q8-Q10,Q11-Q13) расположенные на промежутке инжекции, формируют необходимые амплитудные функции и частоты бетатронных колебаний. Функции фокусировки накопительного кольца «НЕСТОР» [14] показаны на рис.3а,б.

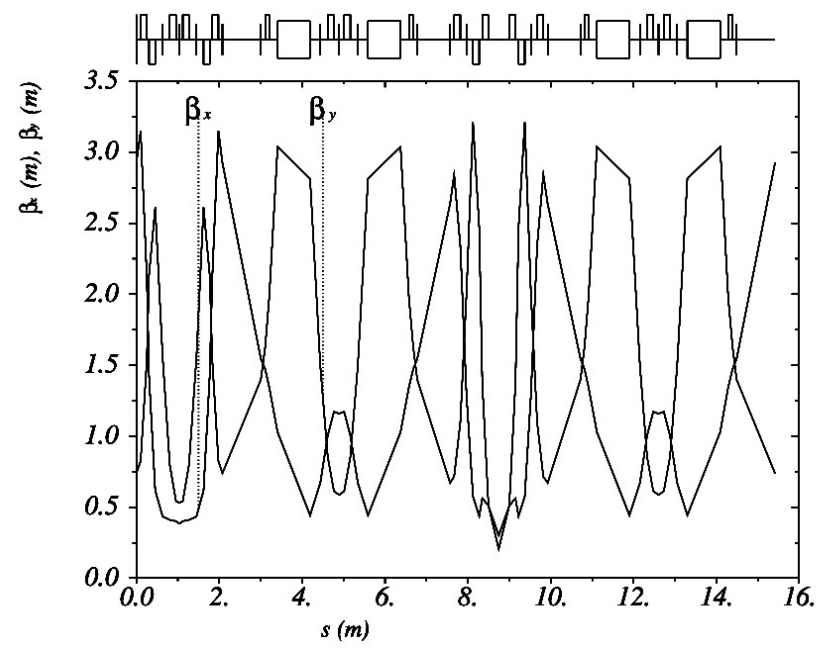

a)

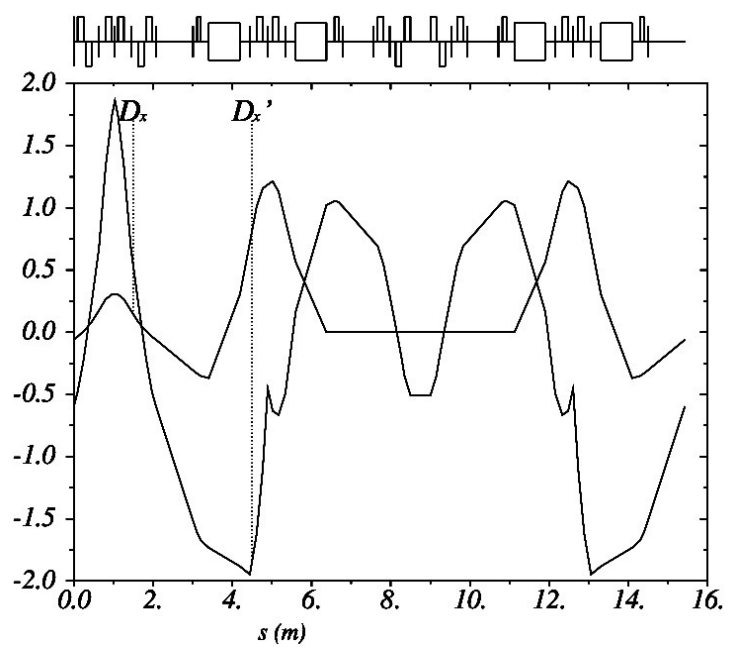

б)

Рис.3. Функции фокусировки накопительного кольца «НЕСТОР»

а) амплитудные функции б) дисперсионные функции

Для подавления хроматических эффектов и коррекции динамической апертуры в накопителе используется 19 секступольных линз (S1-S19). Структура фокусировки накопительного кольца спроектирована, так что бы обеспечить необходимые набеги фаз бетатронных колебаний между секступолями и минимизировать дисперсию второго порядка $\left(\eta_{2}\right)$ в точке взаимодействия.

Проектная структура фокусировки имеет горизонтальную частоту бетатронных колебаний $\mathrm{Q}_{\mathrm{x}}=3,1$, вертикальную частоту $\mathrm{Q}_{\mathrm{z}}=2,15$ и коэффициент упаковки орбит $\alpha=0,0096$. 


\section{МОДЕРНИЗАЦИЯ СТРУКТУРЫ ФОКУСИРОВКИ}

После производства поворотных магнитов для накопителя, была проведена серия измерений их параметров с помощью датчика Холла. Измерения показали, что параметры изготовленных магнитов отличались от проектных, поворотные магниты не только имеют разные показатели спада магнитного поля, но и силы вертикальной фокусировки существенно ниже проектных.

Моделирование показало, что из-за уменьшения вертикальной фокусировки частота вертикальных бетатронных колебаний становится близкой к частоте целого резонанса $\mathrm{Q}=2$, в результате чего вертикальное движение в кольце становится неустойчивым. В таблице 1 приведены силы вертикальной фокусировки (К1) для изготовленных поворотных магнитов. Как видно из таблицы, ослабление вертикальной фокусировки $\Delta \mathrm{K} 1$ составляет приблизительно 5-10\%. В конструкции поворотных магнитов не предусмотрены корректирующие обмотки для К1.

Таблица 1

Сила вертикальной фокусировки в изготовленных поворотных магнитах (проектное значение 2.4).

\begin{tabular}{|c|c|c|c|c|}
\hline $\begin{array}{c}\text { Название поворотного } \\
\text { магнита }\end{array}$ & B1 & B2 & B3 & B4 \\
\hline $\mathrm{K} 1, \mathrm{~m}^{-2}$ & 2,20 & 2,30 & 2,24 & 2,28 \\
\hline
\end{tabular}

Из-за того, что максимально достижимое магнитное поле в поворотных магнитах меньше проектного, максимальная рабочая энергия накопителя уменьшилась с 225 МэВ до 200 МэВ. Максимальная энергия жесткого излучения уменьшилась с 900 КэВ до 720 КэВ [15].

Вторая причина модернизации заключается в необходимости увеличить длину одного из дрейфовых промежутков от 0,45 м до 0,75 м, для установки оптического резонатора.

Для установки дополнительного монитора положения пучка на участке взаимодействия, было решено убрать две симметрично расположенные секступольные линзы (S1, S19) вместо одной из которых и был установлен монитор.

Для моделирования динамики электронного пучка использовались программные пакеты MAD-X [16] и DECA [17]. В результате модернизации промежуток взаимодействия был увеличен до 0,75 м.

Размещение магнитных элементов в накопительном кольце, за исключением триплетов на промежутке взаимодействия (Q1-Q3, Q18-Q20) осталось прежним. Силы квадрупольных линз изменены в пределах 10\% от проектных.

Модернизация проходила в два этапа. На первом этапе модернизации расчет параметров структуры проводился в линейном приближении с использованием программы MAD-X. Полученная структура фокусировки полностью удовлетворяла приведенным выше требованиям. На рис.4а,б приведены амплитудные и дисперсионные функции модифицированной структуры соответственно. Значения частот бетатронных колебаний после модернизации $\mathrm{Q}_{\mathrm{x}}=3,13, \mathrm{Q}_{\mathrm{z}}=1,79$ и коэффициент упаковки орбит $\alpha \approx 0,01$.

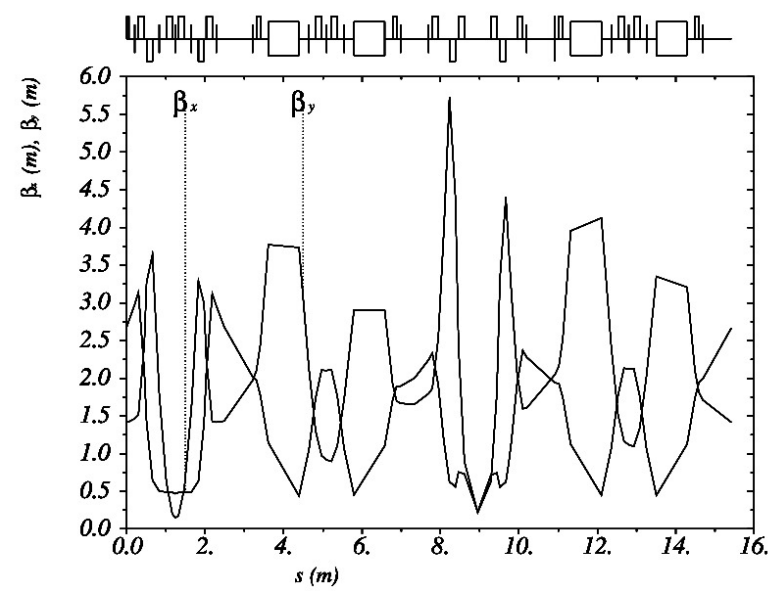

a)

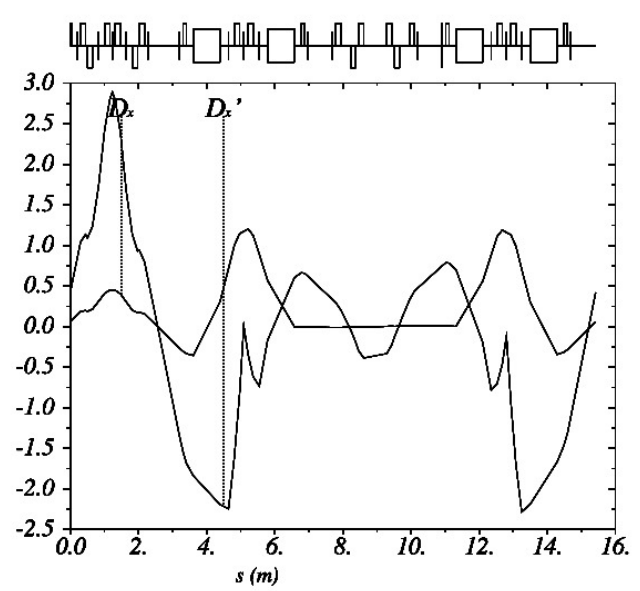

б)

Рис.4. Функции фокусировки накопительного кольца «НЕСТОР» после модернизации а) амплитудные функции б) дисперсионные функции.

На втором этапе модернизации были рассчитаны силы секступольных линз (S1-S17) для подавления естественной хроматичности в накопительном кольце и проведена коррекция динамической апертуры.

После модернизации значение динамической апертуры в точке инжекции составило 38 мм в горизонтальной и 32 мм в вертикальной плоскости, что намного превышает динамическую апертуру проектного накопителя (рис. 5). Увеличенный размер динамической апертуры позволяет более эффективно инжектировать пучок в накопительное кольцо. Основные параметры накопительного кольца НЕСТОР до и после модернизации представлены в таблице 2. 
Зависимость длины электронного сгустка от его энергии приведены на рис.6. Зависимость поперечных размеров электронного сгустка в точке взаимодействия от его энергии приведены на рис.7. С ростом энергии электронного пучка уменьшается влияний эффектов внутрипучкового рассеяния на его поперечные размеры.

Таблица 2

Основные параметры накопительного кольца до и после модернизации

\begin{tabular}{|c|c|c|}
\hline Параметры & До модернизации & После модернизации \\
\hline $\mathrm{Q}_{\mathrm{x}}$ & 3,15 & 3,13 \\
\hline $\mathrm{Q}_{\mathrm{y}}$ & 2,1 & 1,79 \\
\hline$\alpha$ & 0,096 & 0,098 \\
\hline$\beta_{\mathrm{x}}$ в точке взаимодействия & 0,12 & 0,21 \\
\hline$\beta_{\mathrm{y}}$ в точке взаимодействия & 0,14 & 0,23 \\
\hline
\end{tabular}

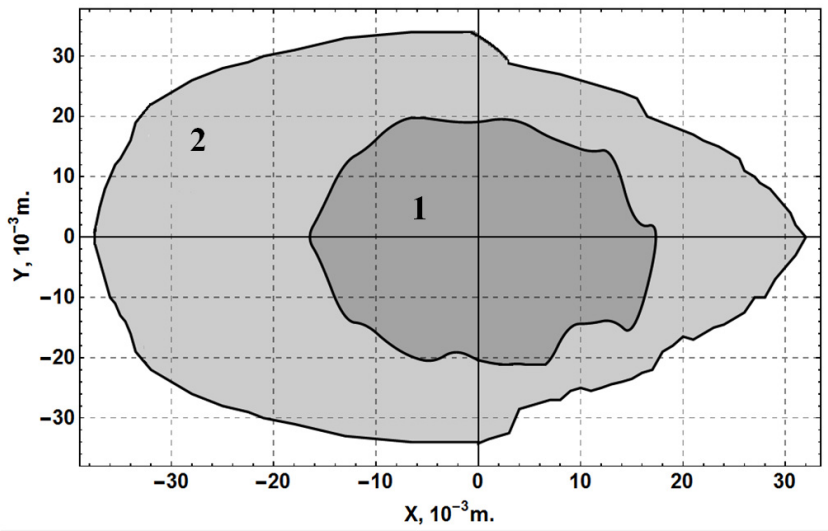

Рис.5: Динамическая апертура в точке инжекции до (1) и после (2) модернизации.

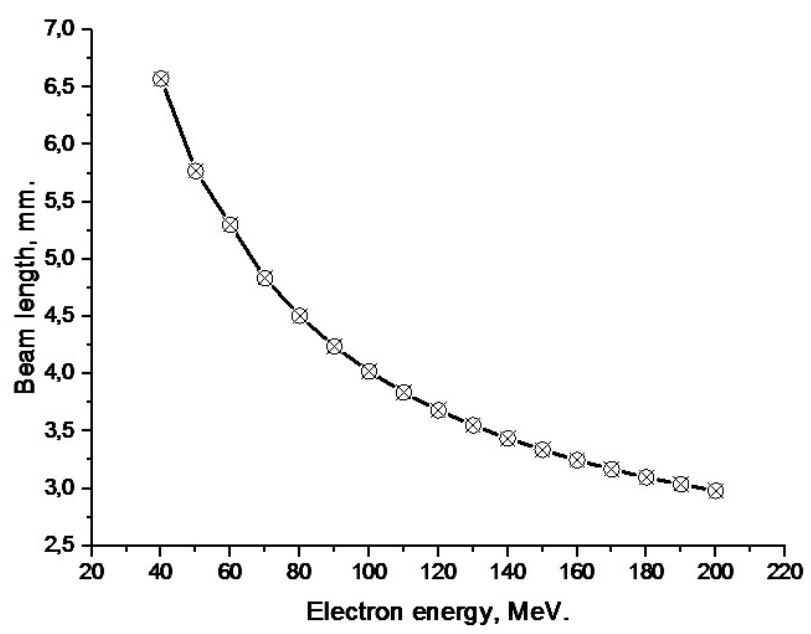

Рис.6: Зависимость продольного размера электронного сгустка от энергии

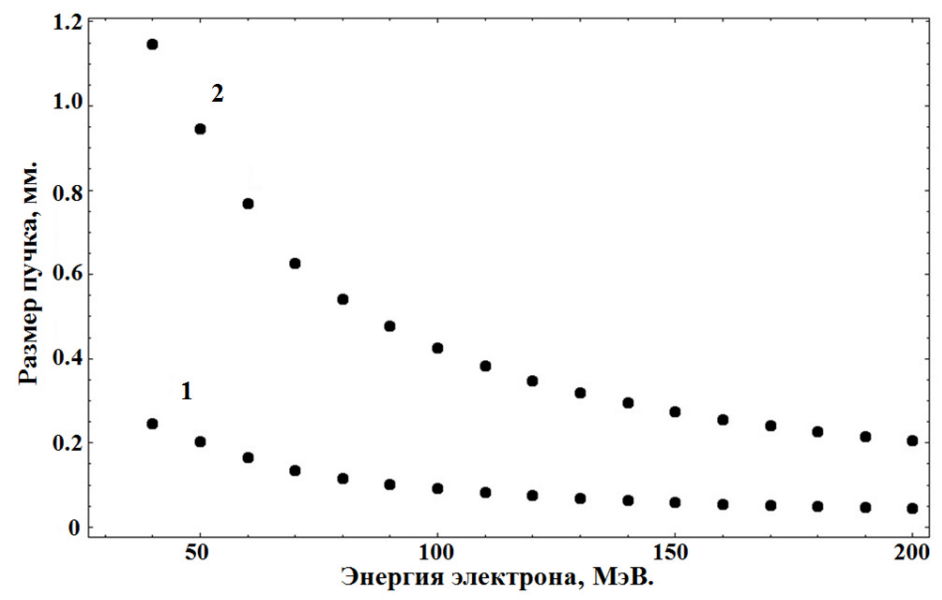

Рис.7: Зависимость поперечных размеров электронного сгустка от энергии 1 - вертикальная плоскость, 2 - горизонтальная плоскость.

Сделаем оценку интенсивности комптоновского рассеяния в накопительном кольце с учетом реальных параметров сталкивающихся пучков:

• Длиной орбиты 15,418 м (частота обращения 19,5 МГц);

- Угол столкновения $\varphi=12^{\circ}$;

- Энергия лазерного фотона $\varepsilon_{1}=1,16$ эВ;

- Частота следования лазерного импульса 350 МГц;

- Средняя мощность лазера $\mathrm{P} \approx 10 \mathrm{BT}$;

- Количество фотонов в одном импульсе $1,5 \times 10^{11}$;

- На орбите накопителя находится 36 электронных сгустков (взаимодействует 18);

• Заряд в одном электронном сгустке 0,5 нКл; 
• Электронный сгусток в точке встречи имеет размеры, приведенные на рис.8 и рис.9; 50 мкм;

- Поперечные размеры лазерного сгустка в вертикальной и горизонтальной плоскости равны $\sigma_{\mathrm{xl}}=\sigma_{\mathrm{zl}}=$

- Продольный размер лазерного сгустка $\sigma_{\mathrm{sl}}=3 \mathrm{Mм;}$

- Коэффициент накопления оптического резонатора 2000.

На рис.8 приведены результаты моделирования зависимости интенсивности комптоновского рассеяния от энергии электронного пучка.

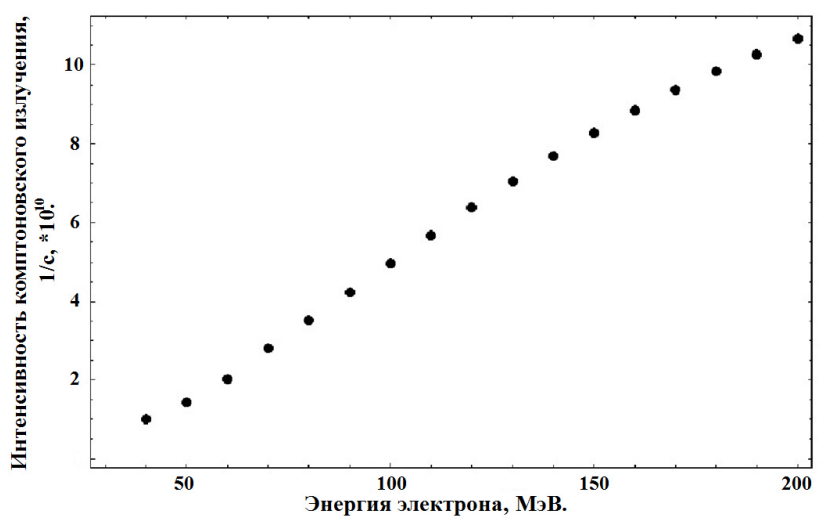

Рис.8: Зависимость интенсивности комптоновского рассеяния от энергии электронного пучка

\section{ВЫВОДЫ}

В результате модернизации структуры фокусировки накопителя НЕСТОР удалось увеличить длину дрейфового промежутка для установки оптического резонатора и решить проблемы, возникшие из-за ошибок изготовления поворотных магнитов. Все основные параметры модернизированной структуры накопительного кольца соответствуют проектным. Удалось значительно увеличить динамическую апертуру, что позволит более эффективно инжектировать электронный пучок в накопительное кольцо.

Оценки показывают, что разработанный источник позволит генерировать жесткое излучение с энергией от 30 КэВ до 720 КэВ и интенсивностью до $10^{11}$ фотонов в секунду.

\section{СПИСОК ЛИТЕРАТУРЫ}

1. Mytsykov A., Kalamayko A, Karnaukhov I. et al. // Modificated lattice of the Compton X-ray source NESTOR // Proceedings of IPAC 2011. - TUPC-040. - P. 1087-1089.

2. Motz H. Applications of the radiation from fast electron beams // Journal of Apply Physics. - 1951. -Vol. 22. - No. 527. P. 527-531.

3. Landecker K. Possibility of frequency multiplication and wave amplification by means of some relativistic effects // Physical Review. - 1952. - Vol. 86. - No.6. - P. 852-855.

4. Artumanyan F., Tumanyan V. Compton effect on relativistic electrons and the possibility of generation of hard radiation // JETP. - 1963. - Vol. 44. - P. 2101.

5. Milburn R. // Thomson Scattering of Optical Radiation from an Electron Beam // Physical Review Letters. - 1963. - Vol. 10. P. 75 .

6. Casano L. et al. Laser scanning components and techniques: design considerations // Laser and Unconventional Optics Journal. - 1975. - Vol. 55. - P. 3.

7. Telnov V. Principles of photon colliders // Nuclear Instruments and Methods in Physics Research A. - 1995. - Vol. 355. P. 3-18.

8. Luo W., Zhuo H. et al. The nonlinear effect in relativistic Compton scattering for an intense circularly polarized laser // Physica Scripta. - 2014. - Vol. 89(7). - P. 5.

9. Huang Z. Radiative cooling of relativistic electron beams // SLAC-R. - 1998. - Vol. 527. - P. 141.

10. Gladkikh P., Karnaukhov I. et. al. Physical Grounding H-100M // Internal report of NSC KIPT. - 1998. - P. 7-30.

11. Liu Lin, Cylon E.T. Goncalves da Silva Second order single particle dynamics in quasi-isochronous storage rings and its application to the LNLS - UVX ring // NIM A. - 1993. - Vol.329. - P. 9-15.

12. Pellegrini C., Robin D. Quasi - isochronous storage ring // NIM A. - 1991. - Vol. 301. - P. 27-36.

13. Zelinsky A., Shcherbakov A. et al. The Kharkov X-ray generator facility NESTOR // Proceedings of IPAC. - 2013. - P. 22532255 .

14. Zelinsky A., Gladkikh P. et al. Investigation of injection through bending magnet fringe fields in X-rays source based on storage ring NESTOR // Proceedings of European Particle Accelerator Conference. - 2004. - P. 1434.

15. Gladkikh P., Karnaukhov I. et al. The start of X-ray generator NESTOR commissioning // Problems of atomic science and technology. -2013. - Vol. 6(88). - P. 20-23.

16. Iselin F. C. The MAD Program. Physical Methods Manual // CERN/SL. -1992.

17. Gladkikh P., Strelkov M., Zelinsky A. The Application Package DECA for Calculating Cyclic Accelerators // Proceedings of PAC-93. - 1993. - P 194-196. 